\title{
Are Genetic Algorithms Really the Best Choice for Building Energy Optimization?
}

\author{
Thomas Wortmann**1, Christoph Waibel ${ }^{* 2,3}$, Giacomo Nannicini ${ }^{1}$, Ralph Evins ${ }^{2,5}$, \\ Thomas Schroepfer ${ }^{1}$, Jan Carmeliet ${ }^{3,4}$ \\ ${ }^{1}$ Architecture and Sustainable \\ Design, SUTD, Singapore \\ ${ }^{4}$ Laboratory for Multiscale \\ Studies in Building Physics, \\ Empa, Duebendorf, Switzerland \\ ${ }^{3}$ Chair of Building Physics, \\ ETH Zurich, Switzerland
}

\begin{abstract}
This paper considers which black-box optimization methods are most appropriate for building energy optimization by revisiting a seminal work concerned with this question. We benchmark three categories of black-box optimization methods - (1) direct search, (2) metaheuristics and (3) model-based methods - on three building energy optimization problems. Considering speed of convergence and stability, we find well-performing methods from all categories, but the widely popular genetic algorithm performs poorly. We also extensively analyze the fitness landscape of one of the three problems. To understand why algorithms fail or succeed, we relate this analysis to the methods' performance. Our results show that the sweeping generalizations on the appropriateness of metaheuristics, and especially genetic algorithms, for building energy optimization require critical scrutiny, while other types of algorithms deserve increased interest in this field.
\end{abstract}

\section{Author Keywords}

Building Energy Optimization, Genetic Algorithms, Direct Search, Model-based Optimization, Fitness Landscape Analysis

\section{ACM Classification Keywords}

I.6.1 Simulation and Modelling, B.5.2 Optimization

\section{INTRODUCTION}

To find energy-efficient building designs, building energy simulation is increasingly combined with black-box optimization methods. This paper considers which blackbox optimization methods are most appropriate for building energy optimization (BEO) by revisiting a seminal work [23] concerned with the same question. Specifically, we ask if genetic algorithms - the most popular algorithm in BEO [5], [15] — should really be the preferred choice.

Black-box (i.e., derivative-free) optimization methods [3, 25] require no knowledge about the mathematical

\footnotetext{
${ }^{*}$ These two authors contributed equally to this work.
}

formulation of an optimization problem, but only consider the problem inputs (variables) and output (cost). The relationship between variables and cost defines a cost function whose range is often referred to as a fitness landscape. In BEO, one usually calculates the cost function with a simulation program such as EnergyPlus.

An important criterion for black-box optimization methods is whether they are local or global. While local methods work best on unimodal fitness landscapes with a single optimum, global methods apply to multimodal problems with multiple optima. The local optima of multimodal problems, which are optimal only relative to a region of the fitness landscape, easily trap local methods. Global methods avoid this entrapment by balancing (global) exploration, which surveys the overall form of the fitness landscape, with (local) exploitation, which finds good solution in promising regions of the landscape.

This study evaluates the performance of optimization methods currently available for Grasshopper-a popular parametric modelling software among architects with links to various simulators-for solving BEO problems. We consider three categories of black-box optimization methods: direct Search, metaheuristics and model-based methods.

\subsection{Direct Search}

Direct search methods are deterministic and sequential. The mathematical optimization community prefers them over metaheuristics due to their proven convergence properties [3] and generally superior performance on (convex, nonconvex, smooth and non-smooth) benchmark problems [19]. There are local and global direct search methods.

\subsection{Metaheuristics}

Metaheuristics [26] often lack proven convergence properties and often draw their motivation from physical and biological phenomena. While many mathematicians view them as methods "of last resort" [3], according to the $\mathrm{BEO}$ literature, metaheuristics are preferable to direct 
search methods. The BEO literature supposes that fitness landscapes in BEO are non-convex, non-smooth and discontinuous [23], and that, due to their stochastic and population-based characteristics, metaheuristics tackle such discontinuous fitness landscape more easily and without getting trapped by local optima[1], [11].

Such arguments are pervasive [5], [14], [15] and next to ease of implementation and availability explain the popularity of metaheuristics. This popularity also applies to structural design optimization and leads [7] to suggest that "evolutionary algorithms may be overused, specifically for continuous problems". In BEO, the rare comparisons between direct search and metaheuristic methods usually feature dated direct search methods like Hooke-Jeeves or Simplex [15].

\subsection{Model-based Methods}

Global model-based methods are a younger category of algorithms. They use machine-learning methods such as Neural Networks, Support Vector Machines and Radial Basis Functions (RBF) to approximate the unknown fitness landscape [12]. The surrogate model replaces or supplements time-intensive simulations since, although less accurate, it is much faster to evaluate. To determine a promising solution to simulate next, the algorithm searches the model deterministically, randomly or with a metaheuristic. The model is then updated with information gained from the simulation.

Model-based algorithms are seldom used for sustainable building design, although Wortmann and Nannicini present promising benchmark results for daylight optimization [25].

Local model-based methods - also known as Trust Region methods [3] - also employ a surrogate model. However, they construct only partial and comparatively simple models. Accordingly, trust region methods work best for convex fitness landscapes without multiple optima.

\section{METHODOLOGY}

This section discusses the considered black-box optimization algorithms, the problems from energy simulation and our benchmarking methodology and criteria.

\subsection{Black-Box Optimization Algorithms}

We compare the performance of nine black-box algorithms, all of which are available for Grasshopper. For the global model-based algorithm, we test two types of surrogate model. Algorithms where no reference is given are covered in [26].

\section{Direct Search Algorithms}

SUBPLEX [20] hybridizes two local direct search algorithms. It aims to improve the performance of the Nelder-Mead Simplex algorithm - one of the algorithms tested in [23] — by decomposing the fitness landscape into smaller regions (i.e., subspaces). It searches individual regions with the Simplex algorithm and moves from one region to a better one with line search. Line search algorithms [3] iteratively select a direction to move to and search for a better solution in that direction.

DIRECT [9] is a global method that considers the whole fitness landscape. It recursively subdivides this landscape into multidimensional hyperrectangles. The algorithm estimates the potential of each rectangle to contain an improved solution and subdivides the most promising one. The result is a grid of solutions that is coarse in unpromising regions and fine in promising ones. DIRECT is rarely applied to building performance optimization, but has shown good performance for structural and daylighting optimization problems [25].

We test the implementations of SUBPLEX and DIRECT from the NLopt library[8], which is linked to Grasshopper via the free Goat plug-in.

\section{Metaheuristic Algorithms}

We consider three "classic" metaheuristics algorithms - a genetic algorithm, particle swarm optimization and simulated annealing - and CRS2, a method which falls inbetween direct search and metaheuristics.

Genetic algorithms evolve a population of good solutions through genetics-inspired operations. Crossover and recombination of individual solutions allow large jumps across the fitness landscape that help to avoid entrapment by local optima, while mutation facilitates gradual changes. Selection ensures the convergence of the population towards a group of good, often very similar, solutions.

Schooling behaviors exhibited by, for example, birds and fish inspired particle swarm optimization (PSO). The swarm's particles represent a population of solutions. A particle moves towards a direction weighted randomly between the best solution encountered by itself and the best solution overall. In this way, the swarm converges gradually in a good region of the landscape, while a broad initial distribution of particles insures against entrapment by local optima.

Simulated annealing (SA) considers only a single solution. Mimicking the movement of an atom in a cooling metal, the solution initially changes more randomly - there is a chance that the solution will get worse-and becomes more stable - only accepting improved solutions - as the "temperature" drops. In other words, this method gradually shifts from exploration to exploitation.

Controlled random search (CRS2) shares similar features with a Nelder-Mead Simplex and is a metaheuristic algorithm in the sense that it heuristically improves a randomly generated population of solutions [10].

We test implementations of a GA and SA that are included in Galapagos, the PSO implementation in Silvereye [2] and the implementation of CRS2 in NLopt linked to by Goat. Galapagos is distributed with Grasshopper, while Silvereye is a free, third-party plug-ins. 


\section{Model-based Algorithms}

COBYLA [17] and BOBYQA [18] are local, trust region algorithms. COBYLA uses a linear and BOBYQA a quadratic model.

We test a global model-based method that interpolates the surrogate model with Radial Basis Functions [6] and a GA to search it. These functions allow different interpolations, of which we test thin-plate spline (RBFtps) and cubic $(\mathrm{RBFc})$, the two best-performing interpolations in [4].

We test the implementation in the open-source RBFOpt library [4], which is linked to Grasshopper via Opossum, a free Grasshopper plug-in.

\subsection{Building Energy Optimization Problems}

We apply the nine optimization algorithms to three building energy design problems using the building energy simulation (BES) program EnergyPlus V8-5-0 linked to Grasshopper via a custom script.

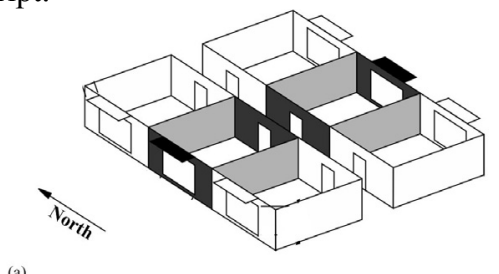

(a)

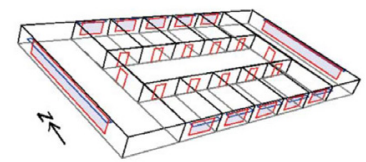

Figure 1. Office buildings used in the numerical experiments: (a) problem 1, (b) problem 2 and 3. Image from: [23]

Problem 1 is a simple office building with four decision variables: building orientation $\alpha$ in $\left[^{\circ}\right]$, window width for the West and East façade $w_{W}$ and $w_{E}$ in [m], and the shading device transmittance $\tau$. Problem 2 is a more detailed office building, where the decision variables control the window width and heights for the North, West, East and South façade $\left(w_{N}, w_{W}, w_{E}, w_{S}\right)$, the depth of the window overhangs in West, East and South $\left(o_{W}, o_{S}, o_{E}\right)$, the setpoint of the shading devices in $\left[\mathrm{W} / \mathrm{m}^{2}\right]$ at the North, East and South façade $\left(s_{W}, s_{E}\right.$, $s_{S}$ ), the setpoint for the zone air temperature for night cooling during summer and winter in $\left[{ }^{\circ} \mathrm{C}\right]\left(T_{u}, T_{i}\right)$, and the cooling design supply air temperature used for the HVAC system sizing in $\left[{ }^{\circ} \mathrm{C}\right]\left(T_{d}\right)$. Problem 3 is identical to Problem 2 with the only difference being the use of discrete variables.

We test problem 1 with Seattle, problem 2 with Houston and problem 3 with Chicago weather. The building models for all problems are shown in Figure 1. Table 1 summarizes the variables, their bounds and their discretization step sizes (form Problem 3). The optimization aims to identify variable values that minimize the annual energy consumption in $\mathrm{kWh} / \mathrm{m}^{2} \mathrm{a}$ for heating, cooling and lighting of the office spaces (i.e., the cost value).
The BEO problems used here originate from a seminal work by Wetter and Wright [23] where a thorough description of the problems including cost functions can be obtained. In our study, we use a different EnergyPlus version than in [23]. We obtained the original files from the authors and used the official EnergyPlus file updater for transitioning to the current version. The weather files are identical to the original study.

In [23], the authors conclude that the hybrid PSO/HookJeeves algorithm finds the best solutions and the simple GA offers faster convergence (i.e. less time-intensive simulations to be run) at a slight decrease in solution quality.

\begin{tabular}{lllll}
\hline \multicolumn{2}{l}{ Variable symbols } & $\boldsymbol{l} \boldsymbol{b}$ & $\boldsymbol{u} \boldsymbol{b}$ & $\boldsymbol{s}$ \\
\hline \multirow{2}{*}{ Simple } & $\boldsymbol{\alpha}$ & -180 & 180 & - \\
model & $\boldsymbol{w}_{\boldsymbol{W}}, \boldsymbol{w}_{\boldsymbol{E}}$ & 0.1 & 5.9 & - \\
& $\boldsymbol{\tau}$ & 0.2 & 0.8 & - \\
\hline & $\boldsymbol{w}_{\boldsymbol{N}}, \boldsymbol{w}_{\boldsymbol{W}}, \boldsymbol{o}_{\boldsymbol{W}}$ & 0 & 1 & 0.05 \\
& $\boldsymbol{w}_{\boldsymbol{S}}, \boldsymbol{o}_{\boldsymbol{S}}$ & 0 & 1 & 0.05 \\
Detailed & $\boldsymbol{w}_{\boldsymbol{E}}, \boldsymbol{o}_{\boldsymbol{E}}$ & 0 & 1 & 0.05 \\
model & $\boldsymbol{s}_{\boldsymbol{W}}, \boldsymbol{s}_{\boldsymbol{E}}, \boldsymbol{s}_{\boldsymbol{S}}$ & 100 & 600 & 25 \\
& $\boldsymbol{T}_{\boldsymbol{u}}, \boldsymbol{T}_{\boldsymbol{i}}$ & 20 & 25 & 0.25 \\
& $\boldsymbol{T}_{\boldsymbol{d}}$ & 23 & 18 & 0.25 \\
\hline
\end{tabular}

Table 1. Variable symbols, lower bound $l b$, upper bound $u b$ and step size $s$

\subsection{Benchmarking}

Following [23], we run Problem 1 for 300 function evaluations (simulations) and Problems 2 and 3 for 500. On an Intel Core i7 $6700 \mathrm{~K}$ CPU with $4.0 \mathrm{GHz}$, one simple simulation takes about 3 seconds and one detailed simulation about 5 seconds.

\section{Algorithm Parameters}

We use default parameters for all algorithms. Although the performance of optimization algorithms, and especially of metaheuristics, can vary greatly with different parameters, finding the best parameters for a specific problem can require many more function evaluations than the actual optimization process itself.Comparing algorithms in this manner reflects a practical situation where little is known about the optimization problem and time constraints limit the number of function evaluations.

The large performance differences between the optimization algorithms presented here suggest that, rather than spending an evaluation budget on tuning an algorithm to a specific problem, one should try two or three different algorithms. Note that the arguments for applying metaheuristics outlined in section 1.2 do not depend on specific parameters.

\section{Performance Criteria}

We assess the algorithms' performance with two criteria: speed of convergence and stability, i.e. quality and reliability. We measure speed of convergence as the best solution found relative to the number of function evaluations - with the computational overhead for the optimization algorithms considered negligible - and stability as the standard deviation of the results from ten optimizations runs per algorithm. 
DIRECT is the most stable algorithm since it is fully deterministic. COBYLA and BOBYQA also are deterministic, but require a starting solution that we assigned randomly.

\section{BENCHMARK RESULTS}

This section presents the benchmarking results for Problems 1, 2 and 3 from section 2.2.

\subsection{Problem 1}

After 300 evaluations of the continuous Problem 1, DIRECT, RBFtps and PSO find mean solutions at 133.0 $\mathrm{kWh} / \mathrm{m}^{2} \mathrm{a}$, with SA and RBFc 0.1 and CRS $0.2 \mathrm{kWh} / \mathrm{m}^{2} \mathrm{a}$ removed (Figure 2). DIRECT, RBFtps and RBFc exhibit early convergence, with PSO and SA catching up at around 150 evaluations. Generally, these better converging algorithms also display high stability; of the six bestperforming algorithms PSO exhibited the largest variation $\left(0.3 \mathrm{kWh} / \mathrm{m}^{2} \mathrm{a}\right)$ and SA one outlier (Figure 3$)$.

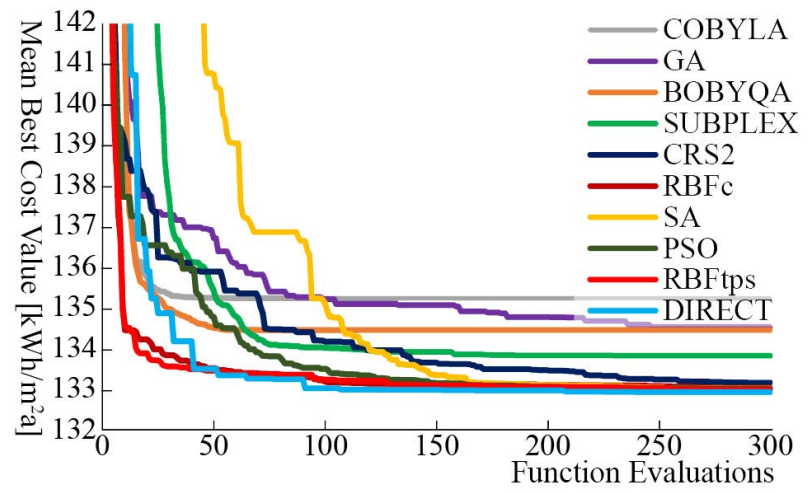

Figure 2. Problem 1: Mean minimum energy consumption from ten runs, as a function of the number of function evaluations.

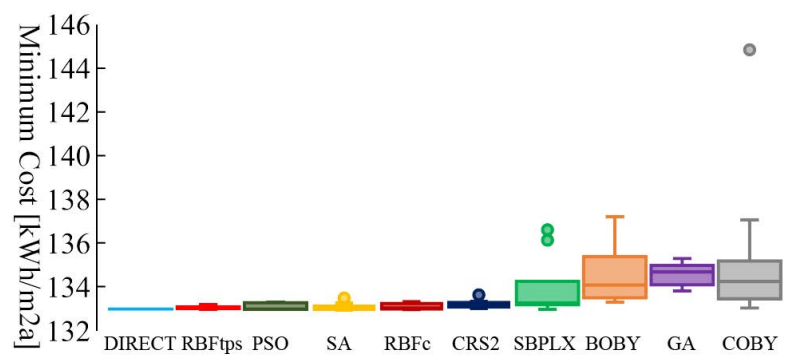

Figure 3. Problem 1: Boxplot of minimum energy consumption after 300 function evaluations from ten runs.

\subsection{Problem 2}

After 500 evaluations of the continuous Problem 2, Subplex finds the best mean solution at $141.3 \mathrm{kWh} / \mathrm{m}^{2} \mathrm{a}$, with DIRECT and SA 0.3, and RBFc and RBDtps $0.5 \mathrm{kWh} / \mathrm{m}^{2} \mathrm{a}$ removed (Figure 4). For the first 200 evaluation, Subplex exhibits the slowest speed of convergence and RBFc and RBDtps the fastest, after which Subplex rapidly improves. Except one dramatic outlier by SA, the better converging algorithms display high stability (Figure 4).

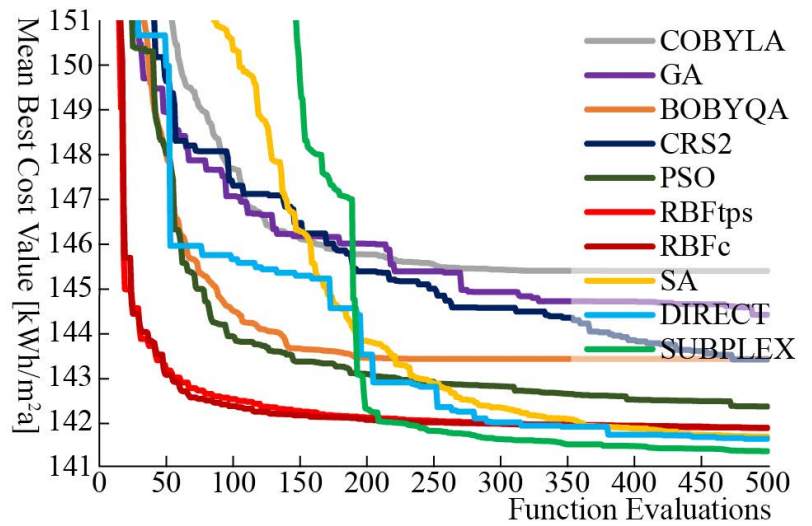

Figure 4. Problem 2: Mean minimum energy consumption from ten runs, as a function of the number of function evaluations.

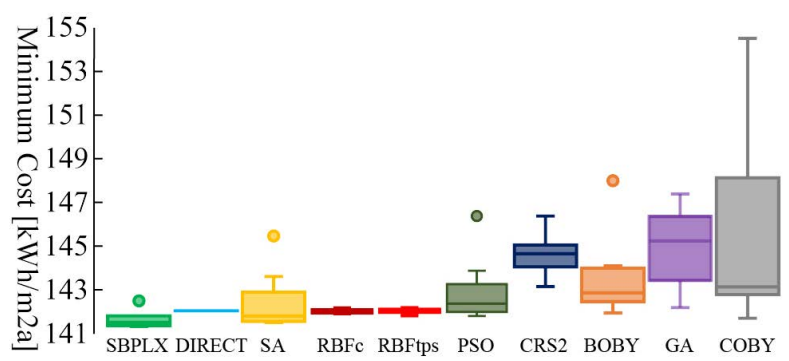

Figure 5. Problem 2: Boxplot of minimum energy consumption after 500 function evaluations from ten runs.

\subsection{Problem 3}

After 500 evaluations of the discrete problem 3, SA finds a solution at $136.0 \mathrm{kWh} / \mathrm{m}^{2} \mathrm{a}$, with DIRECT and RBFc 0.2 and PSO $0.3 \mathrm{kWh} / \mathrm{m}^{2}$ a removed (Figure 6). Initially, RBFc displays the fastest convergence; it is overtaken by SA at around evaluation 150. Problem 3 reveals a large $(0.8$ $\mathrm{kWh} / \mathrm{m}^{2} \mathrm{a}$ ) difference between $\mathrm{RBFc}$ and RBFtpsprobably due to the discretization-which otherwise perform very similarly. Except small outliers by RBFc and PSO, the better converging algorithms display high stability (Figure 7).

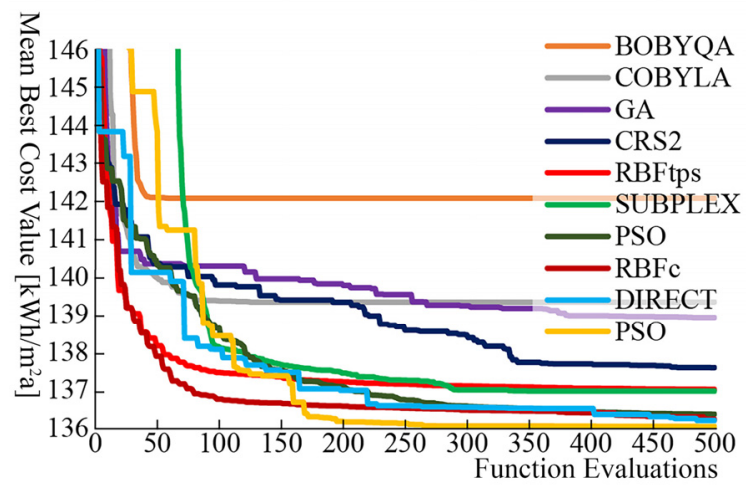

Figure 6. Problem 3: Mean minimum energy consumption from ten runs, as a function of the number of function evaluations. 


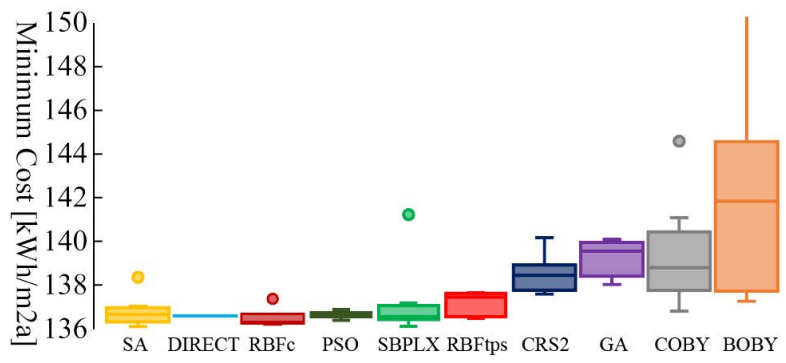

Figure 7. Boxplot of minimum energy consumption after 500 function evaluations from ten runs.

\section{FITNESS LANDSCAPE ANALYSIS}

Using a variety of methods, this section presents a detailed analysis of the fitness landscape for problem 2.

\subsection{Sensitivity Analysis}

We apply the Sobol method, a variance-based sensitivity analysis technique, in order to quantify the amount of variance in the cost value $f(x)$ that can be attributed to changing variable values $x$. The first first-order effect $S_{i}$ indicates the variance in $f(x)$ caused by varying only a single variable $x_{i}$, whereas the total effects $S_{T i}$ indicate the variance in $f(x)$ caused by a variable $x_{i}$ if all other variables are varied as well. Hence, $S_{T i}$ shows the higher-order effects of a variable, i.e. if it interacts with other variables [21]. Both indicators give crucial information about the fitness landscape, as it reveals the most sensitive decision variables in the optimization. For computing $S_{i}$ and $S_{T i}$ we generate a Sobol (also called $\mathrm{LP}_{\mathrm{T}}$ ) sequence, which is a pseudorandom sequence to uniformly distribute samples in a multidimensional hypervolume. The Sobol method is an important technique in BES uncertainty research [13], [22].

$S_{i}$ and $S_{T i}$ for the $n=13$ variables $x_{i} \in\left\{w_{N}, w_{W}, o_{W}, w_{S}, o_{S}\right.$, $\left.w_{E}, o_{E}, s_{W}, s_{E}, s_{S}, T_{u}, T_{i}, T_{d}\right\}$ of problem 2 are shown in Figure 8, using a $\mathrm{LP}_{\mathrm{T}}$ sequence of $m=(n+2) * 1000=$ 15.000 samples. It is striking that only two variables $\left(T_{u}\right.$ and $T_{d}$ ) significantly contribute to the cost $f(x)$, while all other variables appear to be negligible. It is worth noting, however, that the total effects $S_{T i}$ of $T_{u}$ and $T_{d}$ are substantially higher than their first-order effects, indicating a strong interaction between other variables.

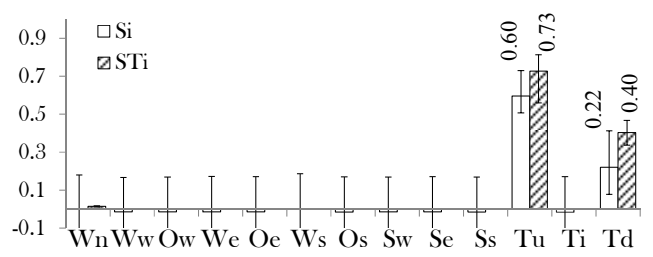

Figure 8. First-order $\left(S_{i}\right)$ and total effects $\left(S_{T i}\right)$ from the Sobol method for problem 2 .

\subsection{Uni- and Bivariate Correlation}

We correlate the $m$ samples from the $\mathrm{LP}_{\mathrm{T}}$ sequence of problem 2 to study the relation of each variable $x_{i}$ to the cost $f(x)$. In the previous section, we identify two major variables, while other variables appear negligible. This impression is confirmed in our correlation plots in Figure 9, where we show the univariate correlation between variables $w_{N}, T_{u}$ and $T_{d}$ over $f(x)$ on the right side of the figure. Variable $w_{N}$ is representative for all other variables (other than $T_{u}$ and $T_{d}$ ), i.e. they show a similar pattern. From the univariate correlations, clear patterns can be recognized for $T_{u}$ and $T_{d}$, while this is less the case for the remaining variables (represented by $w_{N}$ ).

On the left in Figure 9 we show a bivariate correlation of $T_{d}$ and $T_{u}$ over $f(x)$. Each dot is a sample from the $\mathrm{LP}_{\mathrm{T}}$ sequence, i.e. $x[m]$. We overlay a thin plate spline interpolation surface using the Matlab curve fitting toolbox. The importance of the two variables $T_{d}$ and $T_{u}$ becomes especially clear this way, as it shows their joint relation in affecting $f(x)$. Computing the residuals of the samples in relation to the interpolation surface reveals a range between +20 and $-10 \mathrm{kWh} / \mathrm{m}^{2} \mathrm{a}$. In other words, by solely controlling $T_{d}$ and $T_{u}, f(x)$ varies between $\sim 400$ and $\sim 150 \mathrm{kWh} / \mathrm{m}^{2} \mathrm{a}$; by controlling the remaining variables, $f(x)$ can be further varied in the range of +20 and $-10 \mathrm{kWh} / \mathrm{m}^{2} \mathrm{a}$. These last 10 $\mathrm{kWh} / \mathrm{m}^{2} \mathrm{a}$ reduction form the true challenge to be addressed by the optimization algorithms, as indicated in section 3 .
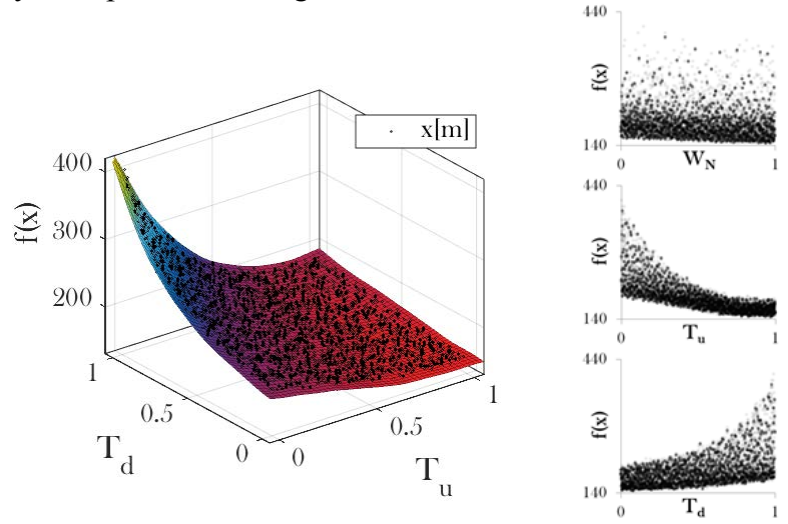

Figure 9. Problem 2. Left: Bivariate correlation between two most sensitive variables $T_{d}$ and $T_{u}$ and cost $f(x)$. Right: Correlation between variables $w_{N}, T_{u}$ and $T_{d}$ and $\operatorname{cost} f(x)$.

\subsection{Fitness Distance Correlation}

Fitness Distance Correlation (FDC) aims to correlate the cost value of a solution and its normalized Euclidean distance in terms of variable space to the global optimum [16]. One of the practical challenges is knowledge about the global optimum $x^{*}$. As a best possible guess, we assume that our global optimum is the best-found solution of all function evaluations from the $\mathrm{LP}_{\mathrm{T}}$ sequence and from all conducted optimization runs. Despite the large sample sizes (15.000 for problem 2) of $\mathrm{LP}_{\mathrm{T}}$ sequences, the best solutions found by optimization algorithms - which typically use a far smaller number of function evaluations (here 500) - are almost always better than the best solutions found by sampling. This efficiency is a strong argument for the need for black-box optimization methods to search design spaces.

While the FDC value indicates how consistently an algorithm is searching the variable space towards the global 
optimum, plotting the distribution of cost $f(x)$ over distance provides insights into the structure of the problem and the behavior of an algorithm.

In Figure 10 we show the FDC plot for problem 2, using the samples generated by the $\mathrm{LP}_{\mathrm{T}}$ sequences. The global optimum lies at 0 on the distance axis. The cost value of the optimum is given in the plot as $f\left(x^{*}\right)$. Furthermore, the best and worst solutions of the $\mathrm{LP}_{\mathrm{T}}$ sequences in terms of cost value $\left(f(x[m])_{\min }\right.$ and $\left.f(x[m])_{\max }\right)$ and distance $\left(d[m]_{\min }\right.$ and $\left.d[m]_{\text {max }}\right)$ are given in the plot. The pattern of problem 2 is highly scattered and does not show any clear lower and upper bounds. It becomes apparent how rugged and nonsmooth the problem landscape is: solutions with similar cost might vary significantly in terms of distance and getting closer to the variables of the best solution might not necessarily improve cost.

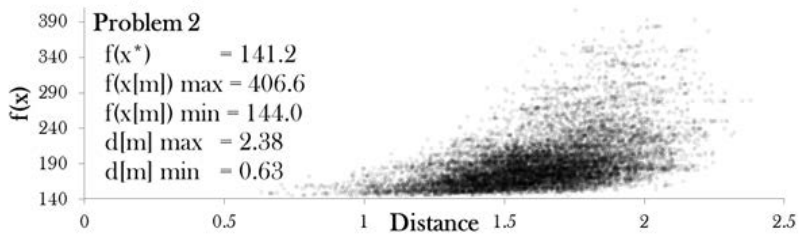

Figure 10. FDC plot of $\mathrm{LP}_{\mathrm{T}}$ sequences for problem 2 .

Figure 11 shows FDC plots for all algorithms and all runs for problem 2. Similar to the convergence graphs, the axes are clipped at $150 \mathrm{kWh} / \mathrm{m}^{2} \mathrm{a}$ and distance 1.5 , since most of the search is conducted in this region. The plots also indicate the FDC values, lowest cost $\left(f_{\text {min }}\right)$ and mean lowest cost of all runs $\left(\bar{f}_{500}\right)$.

The fast convergence of RBFtps and RBFc discussed in section 3 can also be observed here. They invest most of the evaluations to successively reduce the distance towards the best-known value. All runs seem to have a similar search track so that individual runs cannot be identified - proof of the algorithms' stability. PSO shows a similar pattern to $\mathrm{RBF}$, but the search tends to get stuck in local optima. Each run results in discernably different results, indicating a lower stability of this algorithm.

CRS2 shows high randomness of the sample distribution and no consistency in approaching the best-known value. Similarly, GA also shows high randomness, albeit greater convergence to specific regions. But it is unsuccessful in approaching the best-known value and shows very low stability between individual runs.

SA reaches cost values close to the best-known solution, but is unable to approach this solution in terms of variable space: the distance does not fall significantly below 0.5 . While individual search tracks can be seen in the distribution of the samples, SA consistently approaches the same cost value. DIRECT comes close to the best-known value. Its division of the search space into hyperrectangles can be recognized in the distribution of the samples.
The local algorithms SUBPLEX, BOBYQA and COBYLA are easily distinguished in the FDC plots. Individual runs can be tracked by their search paths, especially with BOBYQA. For COBYLA and BOBYQA, this characteristic is fatal. Depending on their starting solutions, they get trapped by local optima and can only refine them. On the other hand, the division into sub-spaces by SUBPLEX allows it to break out of local optima. In this case, it even finds the best value, suggesting that local search can be very effective when started in the right region.

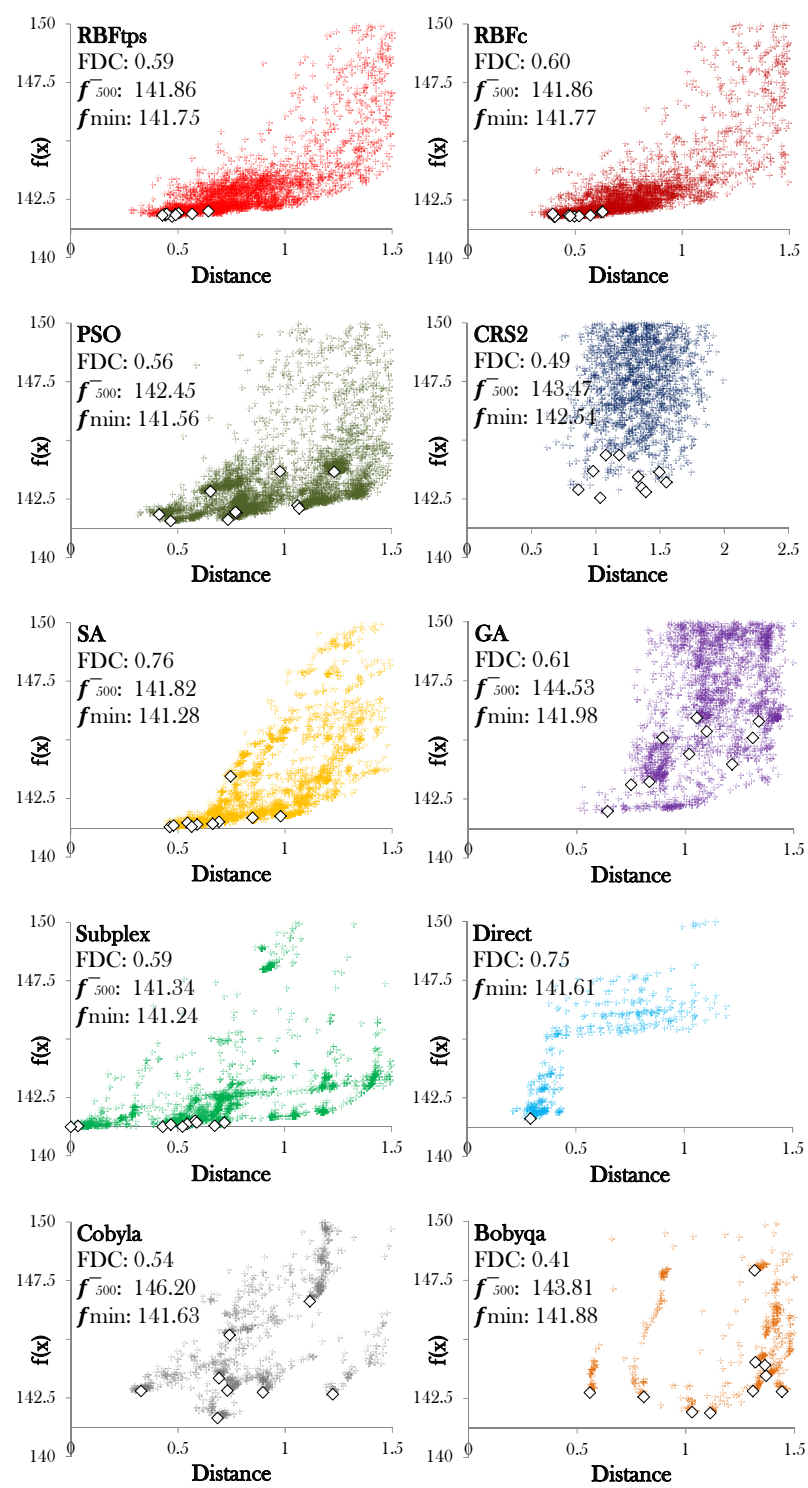

Figure 11. FDC plots of all algorithms for problem 2. The best solution found per run is highlighted as a white diamond shape.

\subsection{Performance Map}

Figure 12 is a "Performance Map" of Problem 2, that-like the FDC plots in Figure 11-visualizes the relationship between variable values and cost. Here, the 13 variables are 
mapped onto the plane by linearly combining variable values with 13 radial coordinate axes: $p=x_{1} v_{1}+\cdots+$ $x_{n} v_{n}$, where $p$ denotes the point location on the plane, $x$ is a variable value, $v$ is its corresponding radial vector, and $n$ is the number of decision variables. Cost is indicated as color with a logarithmic scale. To create the figure, we map all samples (from the optimization runs and the $\mathrm{LP}_{\mathrm{T}}$ sequence), triangulate them and interpolate the colors using barycentric coordinates. [24] covers this method in detail.

The map indicates clusters of very good $\left(\sim 141 \mathrm{kWh} / \mathrm{m}^{2} \mathrm{a}\right)$ and good $\left(\sim 142 \mathrm{kWh} / \mathrm{m}^{2} \mathrm{a}\right)$ solutions. These clusters confirm the problem's multimodality. The location of the clusters in the upper right quadrant of the figure - which is associated with the variables for window size-suggests that better solutions tend to have larger west and east windows and smaller set-points for the shading devices and summer cooling. These better solutions are interspersed with worse ones, which reveals a high degree of discontinuity of the fitness landscape with many local optima.

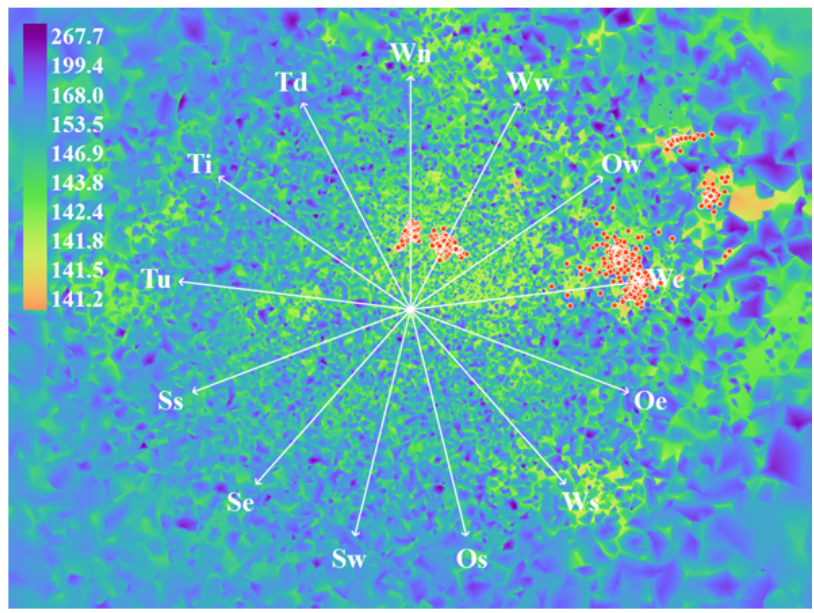

Figure 12. Performance Map of Problem 2. The map visualizes the most relevant portion of the fitness landscape. The red dots indicate solutions within $0.1 \%$ of the best solution.

\section{CONCLUSIONS}

In this benchmark, the best converging algorithms are a global direct search method (DIRECT) on Problem 1, a local global search method (SUBPLEX) on Problem 2 and a metaheuristic (SA) on Problem 3. DIRECT and SA converged fast on other problems as well, although SA is less stable. The RBF algorithms performed slightly less well overall. They excelled in early global exploration, but struggled with exploiting promising local regions. On problems 1 and 3, PSO did well and SUBPLEX struggled, with the reverse being true for problem 2, making these algorithms worth trying. BOBYQA, COBYLA, GA and CRS2 exhibited impractically slow convergence and lower stability than the other algorithms.

Based on the tested implementations, the GA is a poor choice of default algorithm, with DIRECT or SA being better candidates. For problems with a limited number of function evaluations-for example time-intensive daylighting simulations-we recommend RBFc. ([7] and [25] present similar results for structural and daylighting problems.) Since the performance of optimization algorithms is problem-specific, we advise trying several algorithms from different categories when possible.

Sensitivity analysis showed a large impact of two variables for cost reduction and only a small impact by the remaining variables. Nevertheless, their small contributions result in a complex, non-smooth and rugged fitness landscape. This complexity appears in the FDC plots (Figure 11) and performance map (Figure 12). Awareness of a problem's structure helps to understand the performance of optimization algorithms. In this example, algorithms that smooth fitness landscapes (i.e. RBF) or avoid distraction by local optima (i.e. SA, SUBPLEX and DIRECT) successfully balance global exploration and local exploitation.

The authors of [23] assume that local direct search algorithms should perform better on the "rather smooth" Problem 1 and metaheuristics should perform better on Problem 2, because it has "discontinuities (...), which makes optimization with descent algorithms difficult". This hypothesis has not been validated by our results, which do not indicate a clear relationship between the category of algorithm and its performance. Also, only local direct search and no model-based methods were tested in [23].

Compared to [23], we cannot recommend the GA due to its relatively poor performance in our benchmarks. While a direct comparison to [23] cannot be made, mainly since we use different implementations and settings, we can reopen the issue of algorithm choice in the BEO community: The sweeping generalizations one finds in the literature (such as "Evolutionary algorithms are robust in exploring the search space for a wide range of building optimization problems" [1] or "Direct search methods can be very efficient if the objective function doesn't have large discontinuities, otherwise it can fail or get trapped in local minima" [14]) on the appropriateness of metaheuristics for multimodal problems and the limitations of direct search deserve critical scrutiny. In our tests, the GA (a metaheuristic) struggled on all problems, while DIRECT (a direct search method) and RBF (a model-based method) performed well.

One should thus hesitate to generalize the performance of optimization algorithms and not be limited to only one algorithm - or category of algorithm - for a design task, since an algorithm's success depends on the specifics of a problem. The question when to choose which optimization algorithm and with what parameters is of great relevance for designers: There are many algorithms next to the GA and other famous metaheuristics that deserve close attention. 


\section{ACKNOWLEDGMENTS}

The work is related to the Competence Center - Energy and Mobility "Synergistic Energy and Comfort through Urban Resource Effectiveness" project and the Swiss Competence Centers for Energy Research "Future Energy Efficient Buildings and Districts" project.

We thank Michael Wetter and Jonathan Wright for kindly providing us with the original files and for useful discussions. We also thank Georgios Mavromatidis for his kind support on the sensitivity analysis.

\section{REFERENCES}

1. Attia, S., Hamdy, M., O'Brien, W., and Carlucci, S. Assessing gaps and needs for integrating building performance optimization tools in net zero energy buildings design. Energy and Buildings 60, (2013), 110 124.

2. Cichocka, J., Browne, W., and Rodriguez, E. Evolutionary Optimization Processes as Design Tools: Implementation of a Revolutionary Swarm Approach. Proceedings of 31th International PLEA Conference Architecture in (R)Evolution, 2015.

3. Conn, A., Scheinberg, K., and Vicente, L. Introduction to Derivative-Free Optimization. Philadelphia, PA: Society for Industrial and Applied Mathematics, 2009.

4. Costa, A. and Nannicini, G. RBFOpt: an open-source library for black-box optimization with costly function evaluations. Optimization Online 4538, (2014).

5. Evins, R. A review of computational optimisation methods applied to sustainable building design. Renewable and Sustainable Energy Reviews 22, (2013), 230-245.

6. Gutmann, H.-M. A Radial Basis Function Method for Global Optimization. Journal of Global Optimization 19, 3 (2001), 201-227.

7. Hare, W., Nutini, J., and Tesfamariam, S. A survey of nongradient optimization methods in structural engineering. Advances in Engineering Software 59, (2013), 19-28.

8. Johnson, S. G. The NLopt nonlinear-optimization package.

9. Jones, D. R., Perttunen, C. D., and Stuckman, B. E. Lipschitzian optimization without the Lipschitz constant. Journal of Optimization Theory and Applications 79, 1 (1993), 157-181.

10. Kaelo, P. and Ali, M. M. Some Variants of the Controlled Random Search Algorithm for Global Optimization. Journal of Optimization Theory and Applications 130, 2 (2006), 253-264.

11. Kämpf, J. H., Wetter, M., and Robinson, D. A comparison of global optimization algorithms with standard benchmark functions and real-world applications using EnergyPlus. Journal of Building Performance Simulation 3, 2 (2010), 103-120.

12. Koziel, S. and Leifsson, L. Surrogate-Based Modeling and Optimization. Springer New York, 2013.
13. Kristensen, M. H. and Petersen, S. Choosing the appropriate sensitivity analysis method for building energy model-based investigations. Energy and Buildings 130, (2016), 166-176.

14. Machairas, V., Tsangrassoulis, A., and Axarli, K. Algorithms for optimization of building design: A review. Renewable and Sustainable Energy Reviews 31, 1364 (2014), 101-112.

15. Nguyen, A.-T., Reiter, S., and Rigo, P. A review on simulation-based optimization methods applied to building performance analysis. Applied Energy 113, (2014), 10431058.

16. Pitzer, E. and Affenzeller, M. A Comprehensive Survey on Fitness Landscape Analysis. Recent Advances in Intelligent Engineering Systems (2012), 161-191.

17. Powell, M. J. A Direct Search Optimization Method that Models the Objective and Constraint Functions by Linear Interpolation. , in Advances in Optimization and Numerical Analysis, S. Gomez and J.-P. Hennart, Eds. Dordrecht: Springer Netherlands, 1994, 51-67.

18. Powell, M. J. The BOBYQA algorithm for bound constrained optimization without derivatives. University of Cambridge, Cambridge, UK, NA Report NA2009/06.

19. Rios, L. M. and Sahinidis, N. V. Derivative-free optimization: A review of algorithms and comparison of software implementations. Journal of Global Optimization 56, 3 (2013), 1247-1293.

20. Rowan, T. H. Functional Stability Analysis of Numerical Algorithms. Ph.D. Dissertation, The University of Texas, Austin, TX, 1990.

21. Sobol', I. Global sensitivity indices for nonlinear mathematical models and their Monte Carlo estimates. Mathematics and Computers in Simulation 55, 1 (2001), 271-280

22. Tian, W. A review of sensitivity analysis methods in building energy analysis. Renewable and Sustainable Energy Reviews 20, (2013), 411-419.

23. Wetter, M. and Wright, J. A comparison of deterministic and probabilistic optimization algorithms for nonsmooth simulation-based optimization. Building and Environment 39, 8 (2004), 989-999.

24. Wortmann, T. Surveying design spaces with performance maps: A multivariate visualization method for parametric design and architectural design optimization. International Journal of Architectural Computing 15, 1 (2017).

25. Wortmann, T. and Nannicini, G. Black-box optimization for architectural design: An overview and quantitative comparison of metaheuristic, direct search, and modelbased optimization methods. Living Systems and MicroUtopias, (2016), 177-186.

26. Yang, X.-S. Engineering Optimization: An Introduction with Metaheuristic Applications. Hoboken, NJ: John Wiley \& Sons, 2010. 